\title{
$\$$ Research Square \\ Effects Of Intensive Insulin Therapy On The Retinal Microvasculature In Patients With Type 2 Diabetes Mellitus: A Prospective Observational Study
}

\author{
Ning Yang \\ Beijing Tongren Eye Center \\ Ming-Xin Li \\ The Affiliated Hospital of Xuzhou Medical University \\ Xiao-Yan Peng ( $\sim 74000041 @ c c m u . e d u . c n$ ) \\ Beijing Tongren Eye Center
}

\section{Research Article}

Keywords: Retinal microvasculature, Intensive insulin therapy, Optical coherence tomography angiography

Posted Date: February 16th, 2022

DOI: https://doi.org/10.21203/rs.3.rs-1283682/v1

License: (9) (1) This work is licensed under a Creative Commons Attribution 4.0 International License. Read Full License

Version of Record: A version of this preprint was published at BMC Ophthalmology on April 22nd, 2022. See the published version at https://doi.org/10.1186/s12886-022-02397-9. 


\section{Abstract}

Background: We examined the changes in retinal microvasculature in type 2 diabetes mellitus (T2DM) using optical coherence tomography angiography (OCTA) before and after intensive insulin therapy.

Methods: This prospective observational study recruited patients with T2DM and divided them into intensive insulin therapy and oral hypoglycaemic agent groups according to different hypoglycaemic methods. All patients enrolled in this study had diabetes without retinopathy or non-proliferative diabetic retinopathy. OCTA was used in all patients before treatment and at 1, 3, and 6 months after treatment. Changes of vessel density and thickness in the macular and optic disc areas were assessed.

Results: The study included 36 eyes in the intensive insulin therapy group and 36 eyes in the oral hypoglycaemic agent group. During the 6-month follow-up, the funduscopy findings of all patients were stable, without significant progression of retinopathy. One month after treatment, vessel density in the deep capillary plexus (DCP) and peripapillary capillary vessel density (ppVD) were significantly decreased by intensification $(P=0.009,0.000)$. And, ppVD was significantly lower in the intensification group than in the oral agent group $(P=0.000)$. At 3 months after treatment, decreases in vessel density induced by intensification were found in the superficial capillary plexus (SCP), DCP, foveal density in a 300- $\mu \mathrm{m}$-wide region around the foveal avascular area (FD-300), and ppVD $(P=0.032,0.000,0.039,0.000)$. The vessel densities of SCP, DCP, FD-300, and ppVD were significantly lower in the intensification group than that in the oral agent group $(P=0.002,0.004$, $0.039,0.001)$. Six months after treatment, decreases in vessel density by intensification were observed in the DCP and ppVD groups $(P=0.000,0.000)$. The Vessel densities of DCP and ppVD in the intensification group were significantly lower than those in the oral agent group $(P=0.004,0.004)$. In the oral hypoglycaemic agent group, vessel density showed no significant change after treatment.

Conclusions: Insulin-intensive treatment caused a transient reduction in vessel density in both the macular and optic disc areas. Retinal microvasculature monitoring using OCTA is important for patients with type 2 diabetes receiving intensive insulin therapy.

\section{Background}

Diabetic retinopathy (DR) is the most common microvascular complication of diabetes mellitus and one of the major causes of blindness in the working-age population [1]. Chronic high blood glucose levels result in damage to the retinal capillaries and nerves, playing an important role in the occurrence and development of DR.

A number of population-based clinical studies have shown that intensive insulin therapy in patients with type 2 diabetes can help normalise blood glucose control in the short term, thus delaying the progression of DR [2]. Intensive insulin therapy can produce rapid improvements in systemic glucose control and reduce the progression of severe diabetic retinopathy [3]. However, some studies have indicated that intensive insulin therapy can aggravate of diabetic retinopathy at an early stage [4, 5]. Bain. [6] summarized various hypotheses to explain this phenomenon, including the co-synergistic effects of insulin and vascular endothelial growth factor (VEGF) on retinal capillaries, the blood retinal barrier breakdown speculation, and upregulation of VEGF theory, all of which remain tentative and inconclusive. The mechanisms underlying the early deterioration of retinopathy following acute intensive insulin therapy are still unknown. Previous studies have focused on the 
progression of the Early Treatment of Diabetic Retinopathy Study (ETDRS) severity scale and clinically significant diabetic macular oedema (DME). However, there is no information on retinal vessel density and the changes in the different layers of the retinal vascular plexus are unknown.

Optical coherence tomography angiography (OCTA) can quantify retinal vessel density and thickness changes, and has been widely used to evaluate retinal ischaemic diseases [7] and neuroophthalmological diseases [8]. With the development of OCTA, macular microvasculature in DR, including the superficial and deep capillary plexus, can be quantified noninvasively and repeatedly [9].

We aimed to use OCTA to evaluate the vessel density and thickness of the macula and optic disc area at different time points in patients with insulin intensification, and to analyse the effects of insulin intensification on retinal microvascular changes in patients with type 2 diabetes.

\section{Methods}

This prospective study was conducted in accordance with the tenets of the Declaration of Helsinki. The ethics committee of the Affiliated Hospital of Xuzhou Medical University approved the study protocol. All participants provided written informed consent.

\section{Subjects and clinical protocols}

In this study, 77 patients with T2DM were enrolled from May 2020 to February 2021. Fasting blood glucose (FBG) was $\geq 11.1 \mathrm{mmol} / \mathrm{L}$ or $\mathrm{HbA} 1 \mathrm{c} \geq 9.0 \%$. The diagnosis of $\mathrm{T} 2 \mathrm{DM}$ was made by endocrinologists according to the American Diabetes Association diagnostic criteria [10]. Clinical data, including a history of hypertension, hyperlipidaemia, smoking, and renal impairment were recorded using a standardised clinical record form. In our research, hypertension was defined as a blood pressure greater than $140 / 90 \mathrm{mmHg}$, or a self-reported history of hypertension. Hyperlipidaemia was defined as either a total cholesterol level of $6.2 \mathrm{mmol} / \mathrm{L}$ or the use of lipidlowering drugs. Using different hypoglycaemic methods, 39 patients were randomised into the intensive insulin group, and 38 patients were randomised into the oral hypoglycaemic agent group. The diagnosis and classification of DR were confirmed according to the international clinical diabetic retinopathy and diabetic macular oedema disease severity scales [11]. One eye of each participant was included in the research. For patients without clinical DR (NDR), one eye was randomly selected. For patients with non-proliferative diabetic retinopathy (NPDR), the eye with a higher stage was selected. If both eyes were in the same stage, one eye was randomly selected. Patients were excluded if they had any of the following conditions at baseline: 1) proliferative DR or diabetic macular oedema; 2) diseases of the vitreoretinal interface and optic nerve; 3 ) other ocular diseases, including but not limited to age-related macular degeneration, retinal arteriovenous occlusion, retinal choroiditis, uveitis, hereditary macular disease, and other fundus diseases that may lead to structural and morphological changes of the macula; 4) a history of ocular trauma or intraocular surgery; 5) myopia more than -6.00 dioptres or axial length of over $26.50 \mathrm{~mm}$; and 6) failure to obtain high-quality images due to refractive interstitial opacity or poor coordination.

Short-term intensive insulin therapy involves injecting insulin at least three times daily or using continuous subcutaneous insulin infusion. The insulin dosage was adjusted based on the adequate frequency and scientific monitoring of blood glucose levels. Oral hypoglycaemic agents typically use glucose-lowering drugs. 
After blood glucose stabilised, the patients were followed up weekly by telephone. Dosage was adjusted to maintain FBG of 4.4-7.0 mmol/L and non-FBG <10.0 mmol/L [12]. HbA1c levels were monitored every 3 months. All enrolled patients underwent an eye examination, containing visual acuity, intraocular pressure, slitlamp microscopy, dilated fundoscopic examination, and OCTA examination, before treatment and at 1, 3, and 6 months after blood glucose stabilisation. OCTA was used to scan both the macular area of the $6 \times 6$-mm mode and the optic disc area of the $4.5 \times 4.5-\mathrm{mm}$ mode in the two groups.

\section{OCTA image acquisition and analysis}

OCTA scans were performed on all subjects using the Avanti RTVue XR system Quantization 2.0 (Version 2017.1, Optovue Inc. USA). The device uses a split-spectrum amplitude decorrelation angiography algorithm with three dimensional projection artefact removal technique. All enrolled patients underwent OCTA examination in a dark room after pupillary dilation. The vessel density of the macular area was collected in the macular HD $6.0 \times 6.0 \mathrm{~mm}$ scanning mode, and the optic disc area was collected in the HD disc $4.5 \times 4.5$ mm scanning mode. A single OCTA image acquisition consisted of a horizontal scan and a vertical scan to remove eye movement artefacts. The superficial capillary plexus (SCP) is defined as a slab extending from the internal limiting membrane (ILM) to $10 \mu \mathrm{m}$ above the inner plexiform layer (IPL). The DCP is a slab extending 10 $\mu \mathrm{m}$ above the IPL to $10 \mu \mathrm{m}$ below the outer plexiform layer (OPL). Foveal density in a $300 \mu \mathrm{m}$ region around the foveal avascular zone (FD-300) is a parameter demonstrating capillary density from the ILM to the OPL in a 300 $\mu \mathrm{m}$ wide region around the foveal avascular zone (FAZ). Peripapillary vessel density (ppVD) and retinal nerve fibre layer (RNFL) thickness were quantified in the radial peripapillary capillary segment, which was defined as a slab extending from the ILM to the RNFL. The peripapillary area was defined as the area between annular contour lines of $2 \mathrm{~mm}$ and $4 \mathrm{~mm}$ around the disc margin. Vessel density was quantified using the splitspectrum amplitude decorrelation angiography software algorithm. The vessel density is defined as the percentage of signal-positive pixels per total pixel.

To ensure adequate scan quality and comparability, the OCTA scans required an image quality score of $\geq 6$. All images were acquired by a single investigator (NY). The segmentation results of OCTA were manually checked and corrected if the boundary deviated from the right position. The following measurement parameters were quantified: vessel density of the SCP and DCP, the corresponding thickness of fovea and macula, FAZ area, FD300, ppVD, and pRNFL thickness.

\section{Statistical Analysis}

IBM SPSS Statistics (IBM Corporation, Chicago, IL, USA) for Windows (version 26.0) was used for statistical analysis, GraphPad Prism ${ }^{\circledR}$ (GraphPad Software Inc., La Jolla, CA) version 9.0.0 was used to plot graphs, and the Kolmogorov-Smirnov test was used to assess variable normality. One-way ANOVA and Chi-square tests were used to compare baseline clinical data between the two groups. Quantitative data are expressed as mean \pm standard deviation $( \pm s$ ). Repeated measures ANOVA was used for changes in quantitative parameters at baseline and at 1, 3, and 6 months after treatment. Bonferroni's post hoc test was used for correction, and comparisons between groups were performed using the independent samples t-test. Statistical significance was set at $P<0.05$.

\section{Results}




\section{General information}

The study enrolled 77 patients with type 2 diabetes, excluding five patients (three patients had poorly controlled blood glucose levels and two patients did not finish scheduled check-ups within the follow-up stages). Table 1 shows the basic clinical data and blood glucose levels of the two groups. Systemic risk factors, such as hypertension, hyperlipidaemia, and smoking at baseline, were similar between the intensive insulin and oral hypoglycaemic agent groups. Among the patients who reached glycaemic targets, the progress of glucose control, represented by fasting plasma glucose level and $\mathrm{HbA} 1 \mathrm{c}$ did not significantly differ between the two groups during the follow-up period. There were no significant differences in general data at baseline $(P>0.05)$.

Table 1 Basic clinical data of subjects 


\begin{tabular}{|c|c|c|c|}
\hline \multirow[t]{2}{*}{ Characteristics } & Intensive Insulin & Oral Agent $₫ n=36 \rrbracket$ & $P$ values \\
\hline & \multicolumn{3}{|l|}{$\bigotimes n=36 \rrbracket$} \\
\hline Age (years) & $54.3 \pm 5.8$ & $55.5 \pm 6.0$ & $0.40^{*}$ \\
\hline Gender (M/F) & $21 / 15$ & $19 / 17$ & $0.64^{+}$ \\
\hline Duration of diabetes (years) & $3.5 \pm 1.6$ & $3.4 \pm 1.9$ & $0.78^{*}$ \\
\hline SBP $(\mathrm{mmHg})$ & $132.9 \pm 12.8$ & $134.1 \pm 11.7$ & $0.69^{*}$ \\
\hline $\mathrm{DBP}(\mathrm{mmHg})$ & $84.8 \pm 10.8$ & $83.8 \pm 8.7$ & $0.68^{*}$ \\
\hline Hypertension & $12(33.3 \%)$ & $15(41.6 \%)$ & $0.63^{+}$ \\
\hline \multicolumn{4}{|l|}{ FBG (mmol/L) } \\
\hline Pre-treatment & $11.2 \pm 1.9$ & $10.8 \pm 1.5$ & $0.33^{*}$ \\
\hline At $1 \mathrm{M}$ follow-up & $5.6 \pm 0.8$ & $5.5 \pm 0.9$ & $0.65^{\star}$ \\
\hline At 3M follow-up & $5.5 \pm 0.9$ & $6.0 \pm 1.0$ & $0.06^{*}$ \\
\hline At $6 \mathrm{M}$ follow-up & $5.4 \pm 1.0$ & $5.7 \pm 0.9$ & $0.17^{*}$ \\
\hline \multicolumn{4}{|l|}{ HbA1c (\%) } \\
\hline Pre-treatment & $9.7 \pm 1.1$ & $9.9 \pm 1.1$ & $0.60^{*}$ \\
\hline At 3M follow-up & $5.7 \pm 0.8$ & $6.0 \pm 0.7$ & $0.24^{*}$ \\
\hline At $6 \mathrm{M}$ follow-up & $6.0 \pm 0.7$ & $5.8 \pm 0.6$ & $0.25^{\star}$ \\
\hline $\operatorname{CREA}(\mu \mathrm{mol} / \mathrm{L})$ & $67.9 \pm 21.4$ & $74.8 \pm 21.4$ & $0.18^{*}$ \\
\hline UREA (mmol/L) & $5.5 \pm 1.6$ & $5.7 \pm 1.8$ & $0.52^{*}$ \\
\hline eGFR (ml/min) & $105.9 \pm 15.5$ & $112.0 \pm 12.4$ & $0.07^{*}$ \\
\hline $\mathrm{TG}(\mathrm{mmol} / \mathrm{L})$ & $1.8 \pm 0.8$ & $1.6 \pm 0.7$ & $0.36^{*}$ \\
\hline $\mathrm{CHOL}(\mathrm{mmol} / \mathrm{L})$ & $5.3 \pm 1.4$ & $4.9 \pm 1.1$ & $0.11^{*}$ \\
\hline Hyperlipidemia & $12(33.3 \%)$ & $8(22.2 \%)$ & $0.43^{+}$ \\
\hline Smoking & $13(36.1 \%)$ & $10(27.8 \%)$ & $0.61^{+}$ \\
\hline
\end{tabular}

Data are shown as mean \pm standard deviation

${ }^{*} P$ values are from one-way ANOVA

${ }^{+} P$ values are from Pearson Chi-square test 
SBP systolic blood pressure; DBP diastolic blood pressure; FBG fasting blood glucose; HbA1c glycated haemoglobulin; eGFR estimated glomerularfiltrationrate; CHOL Cholesterol; TG triglyceride; NPDR nonproliferative diabetic retinopathy

\section{Changes of vessel density and thickness in the macular area}

During the follow-up period of 6 months after insulin intensification, the funduscopy findings of all patients were stable, without diabetic retinopathy progression. At 1, 3, and 6 months after insulin intensification, both vessel density (VD) and retinal thickness in the macular area were lower than those before insulin intensification (Table 2). Bonferroni's post hoc test showed that both 1 month and 6 months after intensive insulin therapy, DCP-VD was lower than before treatment $(P=0.009,0.000)$. Three months after intensive insulin therapy, SCP-VD, DCP-VD, and FD-300 were lower than those before intensification $(P=0.032,0.000$, and 0.039 , respectively). In the oral hypoglycaemic agent group, there were no significant differences in any macular parameter changes (all $P>0.05$ ).

Longitudinal macular changes in SCP-VD, DCP-VD, FD-300, and retinal thickness in the two groups are shown in Figure 1. At 3 months after treatment, the vessel densities of SCP, DCP, and FD-300 in the intensification group were significantly lower than those in the oral agent's group $(P=0.002,0.004$, and 0.039 , respectively). Six months after treatment, DCP-VD in the intensification group was significantly lower than that in the oral agent group $(P=0.004)$.

Table 2 Changes of vessel density(\%), FAZ area $\left(\mathrm{mm}^{2}\right)$, thickness $(\mu \mathrm{m})$ in macular area in insulin intensive group $(n=36)$

\begin{tabular}{|lllll|}
\hline Macular Parameters & Pre-treatment & \multicolumn{3}{l|}{ After treatment } \\
\cline { 3 - 5 } & & $1 \mathrm{M}$ & $3 \mathrm{M}$ & $6 \mathrm{M}$ \\
\hline SCP-VD & $49.1 \pm 2.8$ & $48.0 \pm 3.1$ & $46.6 \pm 2.7^{*}$ & $48.4 \pm 3.1$ \\
\hline DCP-VD & $51.9 \pm 3.9$ & $47.8 \pm 6.4^{*}$ & $45.9 \pm 5.9^{*}$ & $46.1 \pm 5.5^{*}$ \\
\hline FD-300 & $52.7 \pm 4.0$ & $50.3 \pm 4.6$ & $49.0 \pm 4.7^{*}$ & $50.4 \pm 4.2$ \\
\hline FAZ area & $0.28 \pm 0.06$ & $0.30 \pm 0.05$ & $0.31 \pm 0.06$ & $0.31 \pm 0.06$ \\
\hline Fovea thickness & $251.7 \pm 11.3$ & $250.3 \pm 11.0$ & $248.4 \pm 11.2$ & $249.2 \pm 10.9$ \\
\hline Macular thickness & $283.3 \pm 17.4$ & $282.4 \pm 16.4$ & $281.4 \pm 14.4$ & $282.9 \pm 15.6$ \\
\hline
\end{tabular}

For the insulin intensive group, the significant changes compared with pre-treatment are marked with asterisks.

SCP superficial capillary plexus; DCP deep capillary plexus; FAZ foveal avascular zone; FD-300 foveal density in a $300 \mu \mathrm{m}$ wide region around FAZ; $1 \mathrm{M} 1$ month; 3M 3 months; $6 \mathrm{M} 6$ months

\section{Changes of the peripapillary vessel density and corresponding RNFL thickness}

After insulin intensification, ppVD and pRNFL were decreased at 1, 3, and 6 months compared with pretreatment values (Table 3). According to the Bonferroni correction for multiple analyses, ppVD was lower at 1, 3, 
and 6 months after intensive insulin therapy than before treatment $(P<0.01)$. However, in the oral hypoglycaemic agent group, there were no significant differences in the optic disc parameter changes (all $P>$ 0.05).

Figure 2 shows the longitudinal changes in PPVD and pRNFL in the intensive insulin therapy and oral hypoglycaemic agent groups. At all follow-up periods, there were statistical differences in ppVD between the two groups ( $P=0.000,0.000$, and 0.004 , respectively).

Table 3 Changes of peripapillary vessel density(\%),peripapillary RNFL thickness $(\mu \mathrm{m})$ in insulin intensive group $(n=36)$

\begin{tabular}{|lllll|}
\hline Optic Disc Parameters & Pre-treatment & \multicolumn{4}{l|}{ After treatment } \\
\cline { 3 - 5 } & & $1 \mathrm{M}$ & $3 \mathrm{M}$ & $6 \mathrm{M}$ \\
\hline ppVD & $52.4 \pm 3.3$ & $47.9 \pm 3.3^{\star}$ & $48.6 \pm 4.0^{\star}$ & $49.0 \pm 4.0^{*}$ \\
\hline pRNFL & $112.4 \pm 11.4$ & $108.3 \pm 12.1$ & $107.9 \pm 8.0$ & $108.7 \pm 11.0$ \\
\hline
\end{tabular}

The significant changes compared with pre-treatment are marked with asterisks.

ppVD peripapillary vessel density; pRNFL peripapillary retinal nerve fibre layer

\section{Discussion}

In recent years, a growing number of imaging modalities have been used in the evaluation of DR. Although fundoscopy and fluorescein fundus angiography can intuitively and qualitatively evaluate the changes in retinopathy, they cannot reflect the changes in retinal density at different levels [13]. OCTA is non-invasive and can be repeatedly used to monitor changes in capillary levels [14]. Previous studies $[15,16]$ have shown a decrease in retinal vessel density in preclinical diabetic retinopathy. Some studies have also shown [17] that retinal capillary damage in early diabetic retinopathy does not completely form an obvious local non-perfusion area but represents decreased retinal vessel density. Thus, OCTA has a unique value for monitoring DR.

To the best of our knowledge, this study is the first to use OCTA to investigate the effects of insulin intensification on different retinal levels in patients with type 2 diabetes. Quantitative follow-up was conducted to observe the vessel density changes in the macular and optic disc areas and thickness parameters before and after the intensification of insulin treatment. To evaluate the degree of damage to the retinal capillary terminal, it is more accurate to detect changes in the microvascular structure before and after insulin treatment. Previous studies on the effect of insulin intensification of the retina have focused on diabetic retinopathy scales or DME $[6,18]$; however, no studies on microvessel density using OCTA have been conducted. Therefore, it is not clear whether insulin intensification affects NDR and NPDR at a relatively early stage. In our study, we found that insulin intensification caused decreased vessel density in the macular area, which decreased in the first month, decreased significantly at 3 months, and recovered at 6 months after treatment, but was still below the baseline level before intensification. In this study, 3 months after intensive insulin treatment, SCP, DCP, and FD300 were significantly reduced after intensive insulin treatment, and the differences were statistically significant. Macular microvessel density was also statistically different from that of oral hypoglycaemic agents 
during the same period. The results showed that insulin intensification caused the most severe damage to the microcirculation system of superficial and deep capillaries of the retina at three months, leading to ischaemic changes in the retina. The decrease in vessel density in the macular area was most significant 3 months after intensification, but not earlier, and the reasons and mechanisms need to be further studied.

Interestingly, at 1 month after intensification, the vessel densities of both SCP and FD-300 were slightly lower than those before treatment. However, the vessel density of the DCP group was significantly lower than that before treatment. Our findings showed that the early stage of intensive insulin therapy leads to negative effects on the macular microvasculature, especially in the deep capillary plexus. One possible explanation for the preferential involvement of DCP ischaemic changes is that DCP might be more sensitive to insulin intensification due to relatively lower blood flow in the deep capillary plexus [19, 20]. Histopathological studies have shown that diabetic microhaemangiomas originate mainly from the deeper capillary plexus [21], which indirectly proves that deep tissues are more susceptible to hypoxia. SCP mitigated hypoxic damage because it was directly connected to the retinal arterioles, which have a higher perfusion pressure and oxygen supply. These results are consistent with those of previous studies [22, 23]. Our study not only supports earlier results but also adds new longitudinal evidence to previous cross-sectional observations regarding the unique value of microvascular changes in early DCP in diabetic patients [24].

It should be noted that in this study, although the FAZ area increased after intensification, the difference was not statistically significant, considering that there is a large variation in the FAZ area of normal human eyes [25]. Our study not only supported but also added new longitudinal evidence to previous observations [26] that the FAZ area of DR patients was larger than that of normal subjects. We also found that the thickness of the macular area during this period was also lower than that before intensification, indicating that structural damage may be related to the decrease in vessel density, but the difference in the sequence of intensification was not statistically significant.

From the perspective of longitudinal research, we found that retinal microcirculation was extensively affected after insulin intensification. The vessel density and thickness not only changed in the macular area but also decreased in the optic disc area. Microcirculation around the optic disc was the most obvious, and the radial peripapillary vessel density decreased significantly. It is speculated [27] that when vessel density decreases in the macular area at the capillary terminal of the blood supply in ischaemic retinopathy, ppVD upstream of the blood supply may also decrease. The capillaries in the inner layer of the retina continuously branch from the central retinal artery running from the centre of the optic disc. The results of this study are consistent with the above theoretical speculation; at 1 month after treatment, ppVD was statistically lower than that before treatment, while SCP-VD and FD-300 were not significantly changed until 3 months after treatment. This suggests that ppVD is similar to DCP-VD and reflects disease progression earlier than other indicators, suggesting that insulin intensification could cause microcirculation disorder of the optic disc area while early worsening of diabetic retinopathy (EWDR) progresses much earlier.

Radial peripapillary capillaries are the innermost layer of capillaries that run parallel to the peripapillary RNFL and act as a nourish RNFL in its distribution around the optic nerve head. Frydkjaer-Olsen et al. [27] suggested that retinal vessel calibre was independently associated with structural changes of the neuroretina in patients with no or early DR prior to microangiopathy; however, there was no statistically significant decrease in peripapillary RNFL thickness after treatment, and there was a trend of decline. A possible reason is that after 
ganglion cell injury, progressive dendrite contraction first occurs, followed by the disappearance of the cell body and axon; therefore, the reduction of ppVD may occur earlier than the thinning of RNFL thickness. Relevant literature indicates that compared with the normal control group, the decrease in ppVD in the whole week and in each quadrant of the DM group was statistically different, while only part of the quadrants showed statistically significant differences in RNFL decline [28]. Therefore, it was speculated that the decline of ppVD in DM patients might occur before the decline of RNFL, which is consistent with the results of this study. Our study not only supports but also adds new longitudinal evidence to previous cross-sectional observations [29] that monitoring RPC vessel density in the pericapillary region using OCTA may reveal neurodegeneration in the clinical stage of DR. The addition of medications that improve retinal perfusion or optic nerve protection may slow the progression of optic nerve injury [30].

One might argue that a rapid decrease in blood glucose levels results in a transient decrease in retinal microcirculation. However, in the oral hypoglycaemic agent group, there was no significant change in vessel density in the macular and optic disc areas after blood glucose decreased. This suggests that the decrease in vessel density was not due to fluctuations in blood glucose levels. The mechanism underlying decreased retinal vessel density after insulin intensification in patients with type 2 diabetes mellitus is unclear. Studies have shown [31-33] that insulin-like growth factor (IGF-1) can affect the function of retinal endothelial progenitor cells under hypoxia and promote retinal angiogenesis, which plays an important role in the occurrence and development of DR and causes EWDR.

There is no doubt that intensive treatment for glucose compliance can provide long-term benefits for most patients with type 2 diabetes [34]. However, retinal microvascular changes occur in the early stages of intensive treatment, indicating ischaemia. The worsening of diabetic retinopathy manifests prior to the long-term benefits of optimising glycaemic control. Fundus conditions should be closely monitored in the early stage of intensive treatment. Furthermore, chronic kidney disease (CKD), one of the most common complications of diabetes, has a pathogenesis similar to that of DR [35]. Previous studies have shown that renal impairment is related to a reduced retinal capillary density index $[36,37]$. In our study, we found that intensive insulin therapy reduced the retinal microvascular density. We hypothesised that intensive insulin therapy might also affect renal

microvasculature/function. CKD in a patient with DM can be a true diabetic kidney disease (DKD), distinguished only by kidney biopsy [38]. Changes in the retinal microvasculature, which are accessible for direct non-invasive visualisation, may be used to probe the state of systemic microcirculation [39]. Therefore, the next step is a longitudinal investigation of whether there is any influence on renal indicators during intensive insulin therapy. If this is the case, further studies are needed to determine whether non-invasive OCTA imaging can be used to probe the state of renal microcirculation/function.

The strengths of our study include its prospective study design, longitudinal OCTA follow-up, and high-quality data collection. This study has several limitations. First, the quality of the included samples was limited, so we could not determine the effect of different types of insulin on retinal microvascular density. Second, we only investigated the effects of intensive treatment on retinal microvessels, and choroidal vessel density should also be studied using more advanced OCTA machines. Third, only the macular $(6.0 \times 6.0 \mathrm{~mm})$ and optic disc $(4.5 \times$ $4.5 \mathrm{~mm}$ ) areas were involved, which could not reflect the blood perfusion measurement in a wider range, while the lesions site of DR could be located in the whole retina. Therefore, quantitative studies with a larger scanning range should be included in future studies. 


\section{Conclusions}

During the 6-month follow-up period after insulin intensification, retinal vessel density decreased in the SCP, DCP, FD-300, and peripapillary capillaries. Our findings provide new insights into the changes in retinal microvessels before and after insulin intensification. Fundus conditions should be closely monitored during the early stage of insulin-intensive treatment. Monitoring of retinal microvasculature using OCTA is of great importance for patients with type 2 diabetes who are receiving intensive insulin therapy.

\section{Abbreviations}

ANOVA: Analysis of variance; $\mathrm{CHOL}$ : Cholesterol;

CKD: chronic kidney disease; DKD: diabetic kidney disease;

DBP: diastolic blood pressure; DCP: Deep capillary plexus;

DME: diabetic macular oedema; DR: diabetic retinopathy;

eGFR: estimated glomerular filtration rate; ETDRS: Early Treatment of Diabetic Retinopathy Study

FBG: fast blood glucose; FAZ: foveal avascular zone;

FD-300: foveal density in a $300 \mu \mathrm{m}$ wide region around FAZ; HbA1c: glycated haemoglobulin A1c;

NDR: without clinical DR; NPDR: nonproliferative diabetic retinopathy

ILM: internal limiting membrane; SCP: Superficial capillary plexus;

OCTA: Optical coherence tomography angiography; OPL: outer plexiform layer;

PDR: proliferative diabetic retinopathy; pRNFL: peripapillary retinal nerve fibre layer;

ppVD: peripapillary capillaries vessel density; SBP: systolic blood pressure;

T2DM: type 2 diabetes mellitus; TG: triglyceride;

VD: Vessel density; VEGF: vascular endothelial growth factor;

\section{Declarations}

\section{Acknowledgements}

Not applicable.

\section{Authors' contributions}

NY and XP designed the study. NY and ML collected and analyzed all data. XP, NY, and ML interpreted the data and results. NY and $M L$ drafted the manuscript and figures. XP and $M L$ reviewed the manuscript. All authors read and approved the final manuscript. 


\section{Funding}

Not applicable.

\section{Availability of data and materials}

All data generated or analysed during this study are included in this published article and its supplementary information files.

\section{Ethics approval and consent to participate}

The study was in compliance with the tenets of the Declaration of Helsinki. The Ethics Committee of Xuzhou Medical University Affiliated Hospital approved the study protocol. Written informed consent was obtained from all participants.

\section{Consent for publication}

Not applicable.

\section{Competing interests}

The authors declare that they have no competing interests.

\section{Author details}

${ }^{1}$ Beijing Institute of Ophthalmology, Beijing Ophthalmology and Visual Science Key Laboratory, Beijing Tongren Eye Center, Beijing Tongren Hospital, Capital Medical University, NO. 17 Hougou Lane, Chongnei Street, Beijing 100005, People's Republic of China. ${ }^{2}$ Department of Ophthalmology, The Affiliated Hospital of Xuzhou Medical University, 99 West Huaihai Rd, Quanshan District, Xuzhou 221002 Jiangsu, People's Republic of China.

\section{References}

1. Cheloni R, Gandolfi SA, Signorelli C et al. Global prevalence of diabetic retinopathy: protocol for a systematic review and meta-analysis. BMJ Open 2019; 9:e022188.

2. Zoungas $\mathrm{S}$, Arima $\mathrm{H}$, Gerstein $\mathrm{HC}$ et al. Effects of intensive glucose control on microvascular outcomes in patients with type 2 diabetes: a meta-analysis of individual participant data from randomised controlled trials. The Lancet Diabetes \& Endocrinology 2017; 5:431-437.

3. Pettitt DJ, Wollitzer AO, Jovanovic L et al. Decreasing the risk of diabetic retinopathy in a study of case management. Diabetes Care 2005; 28:2819-2822.

4. van B, E., Hooymans J, Timmerman Z et al. Rapid deterioration of diabetic retinopathy during treatment with continuous subcutaneous insulin infusion. Diabetes Care 1984; 7:236-242.

5. Group TDCaCTR. Early worsening of diabetic retinopathy in the Diabetes Control and Complications Trial. Arch Ophthalmol 1998; 116:874-886. 
6. Bain SC, Klufas MA, Ho A et al. Worsening of diabetic retinopathy with rapid improvement in systemic glucose control: A review. Diabetes Obes Metab 2019; 21:454-466.

7. Li X, Yu Y, Liu X et al. Quantitative analysis of retinal vessel density and thickness changes in diabetes mellitus evaluated using optical coherence tomography angiography: a cross-sectional study. BMC Ophthalmol 2021; 21:259.

8. Mammo Z, Heisler M, Balaratnasingam C et al. Quantitative Optical Coherence Tomography Angiography of Radial Peripapillary Capillaries in Glaucoma, Glaucoma Suspect, and Normal Eyes. Am J Ophthalmol 2016; 170:41-49.

9. Sun Z, Yang D, Tang Z et al. Optical coherence tomography angiography in diabetic retinopathy: an updated review. Eye (Lond) 2021; 35:149-161.

10. American Diabetes A. Diagnosis and classification of diabetes mellitus. Diabetes Care 2014; 37 Suppl $1:$ S81-90.

11. Wilkinson CP, Ferris FL, Klein RE et al. Proposed international clinical diabetic retinopathy and diabetic macular edema disease severity scales. Ophthalmology 2003; 110:1677-1682.

12. Umpierrez GE, Hellman R, Korytkowski MT et al. Management of hyperglycemia in hospitalized patients in non-critical care setting: an endocrine society clinical practice guideline. J Clin Endocrinol Metab 2012; 97:1638.

13. Li X, Xie J, Zhang L et al. Differential distribution of manifest lesions in diabetic retinopathy by fundus fluorescein angiography and fundus photography. BMC Ophthalmol 2020; 20:471.

14. Spaide RF, Fujimoto JG, Waheed NK et al. Optical coherence tomography angiography.Prog Retin Eye Res 2018; 64:1-55.

15. Cao D, Yang D, Huang Z et al. Optical coherence tomography angiography discerns preclinical diabetic retinopathy in eyes of patients with type 2 diabetes without clinical diabetic retinopathy. Acta Diabetol 2018; 55:469-477.

16. Dimitrova G, Chihara E, Takahashi H et al. Quantitative Retinal Optical Coherence Tomography Angiography in Patients With Diabetes Without Diabetic Retinopathy. Invest Ophthalmol Vis Sci 2017; 58:190-196.

17. Nesper PL, Roberts PK, Onishi AC et al. Quantifying Microvascular Abnormalities With Increasing Severity of Diabetic Retinopathy Using Optical Coherence Tomography Angiography. Invest Ophthalmol Vis Sci 2017; 58:BI0307-BI0315.

18. Lam PY, Chow SC, Lam WC et al. Management of Patients with Newly Diagnosed Diabetic Mellitus: Ophthalmologic Outcomes in Intensive versus Conventional Glycemic Control. Clin Ophthalmol 2021; 15:27672785.

19. Sun Z, Tang F, Wong R et al. OCT Angiography Metrics Predict Progression of Diabetic Retinopathy and Development of Diabetic Macular Edema: A Prospective Study. Ophthalmology 2019; 126:1675-1684. 
20. Kim AY, Chu Z, Shahidzadeh A et al. Quantifying Microvascular Density and Morphology in Diabetic Retinopathy Using Spectral-Domain Optical Coherence Tomography Angiography. Invest Ophthalmol Vis Sci 2016; 57:0CT362-370.

21. Horii T, Murakami T, Nishijima K et al. Optical coherence tomographic characteristics of microaneurysms in diabetic retinopathy. Am J Ophthalmol 2010; 150:840-848.

22. Simonett JM, Scarinci F, Picconi F et al. Early microvascular retinal changes in optical coherence tomography angiography in patients with type 1 diabetes mellitus. Acta Ophthalmol 2017; 95:e751-e755.

23. Onishi AC, Nesper PL, Roberts PK et al. Importance of Considering the Middle Capillary Plexus on OCT Angiography in Diabetic Retinopathy. Invest Ophthalmol Vis Sci 2018; 59:2167-2176.

24. Karst S, Salas M, Hafner J et al. Three dimensional analysis of retinal microaneurysms. Retina 2019; 39:465-472.

25. Yu J, Jiang C, Wang $X$ et al. Macular perfusion in healthy Chinese: an optical coherence tomography angiogram study. Invest Ophthalmol Vis Sci 2015; 56:3212-3217.

26. Di G, Weihong Y, Xiao Z et al. A morphological study of the foveal avascular zone in patients with diabetes mellitus using optical coherence tomography angiography. Graefes Arch Clin Exp Ophthalmol 2016; 254:873879.

27. Frydkjaer-Olsen U, Soegaard Hansen R, Simo R et al. Correlation between Retinal Vessel Calibre and Neurodegeneration in Patients with Type 2 Diabetes Mellitus in the European Consortium for the Early Treatment of Diabetic Retinopathy (EUROCONDOR). Ophthalmic Res 2016; 56:10-16.

28. Cao D, Yang D, Yu H et al. Optic nerve head perfusion changes preceding peripapillary retinal nerve fibre layer thinning in preclinical diabetic retinopathy. Clin Exp Ophthalmol 2019; 47:219-225.

29. Cheung CY, Chen D, Wong TY et al. Determinants of quantitative optic nerve measurements using spectral domain optical coherence tomography in a population-based sample of non-glaucomatous subjects. Invest Ophthalmol Vis Sci 2011; 52:9629-9635.

30. Hernandez C, Simo R. Neuroprotection in diabetic retinopathy. Curr Diab Rep 2012; 12:329-337.

31. Raman P, Singal AK, Behl A. Effect of Insulin-Like Growth Factor-1 on Diabetic Retinopathy in Pubertal Age Patients With Type 1 Diabetes. Asia Pac J Ophthalmol (Phila) 2019; 8:319-323.

32. Kondo T, Vicent D, Suzuma K et al. Knockout of insulin and IGF-1 receptors on vascular endothelial cells protects against retinal neovascularization. Journal of Clinical Investigation 2003; 111:1835-1842.

33. Chantelau E, Meyer-Schwickerath R, Klabe K. Downregulation of serum IGF-1 for treatment of early worsening of diabetic retinopathy: a long-term follow-up of two cases. Ophthalmologica 2010; 224:243-246.

34. Li Y, Xu W, Liao Z et al. Induction of long-term glycemic control in newly diagnosed type 2 diabetic patients is associated with improvement of beta-cell function. Diabetes Care 2004; 27:2597-2602. 
35. Barrett EJ, Liu Z, Khamaisi M et al. Diabetic Microvascular Disease: An Endocrine Society Scientific Statement. J Clin Endocrinol Metab 2017; 102:4343-4410.

36. Zhuang X, Cao D, Yang D et al. Association of diabetic retinopathy and diabetic macular oedema with renal function in southern Chinese patients with type 2 diabetes mellitus: a single-centre observational study. BMJ Open 2019; 9.

37. Ting DSW, Tan GSW, Agrawal R et al. Optical Coherence Tomographic Angiography in Type 2 Diabetes and Diabetic Retinopathy. JAMA Ophthalmology 2017; 135.

38. Anders HJ, Huber TB, Isermann B et al. CKD in diabetes: diabetic kidney disease versus nondiabetic kidney disease. Nat Rev Nephrol 2018; 14:361-377.

39. Hsieh YT, Hsieh MC. Time-sequential correlations between diabetic kidney disease and diabetic retinopathy in type 2 diabetes - an 8-year prospective cohort study. Acta Ophthalmol 2021; 99:e1-e6.

\section{Figures}
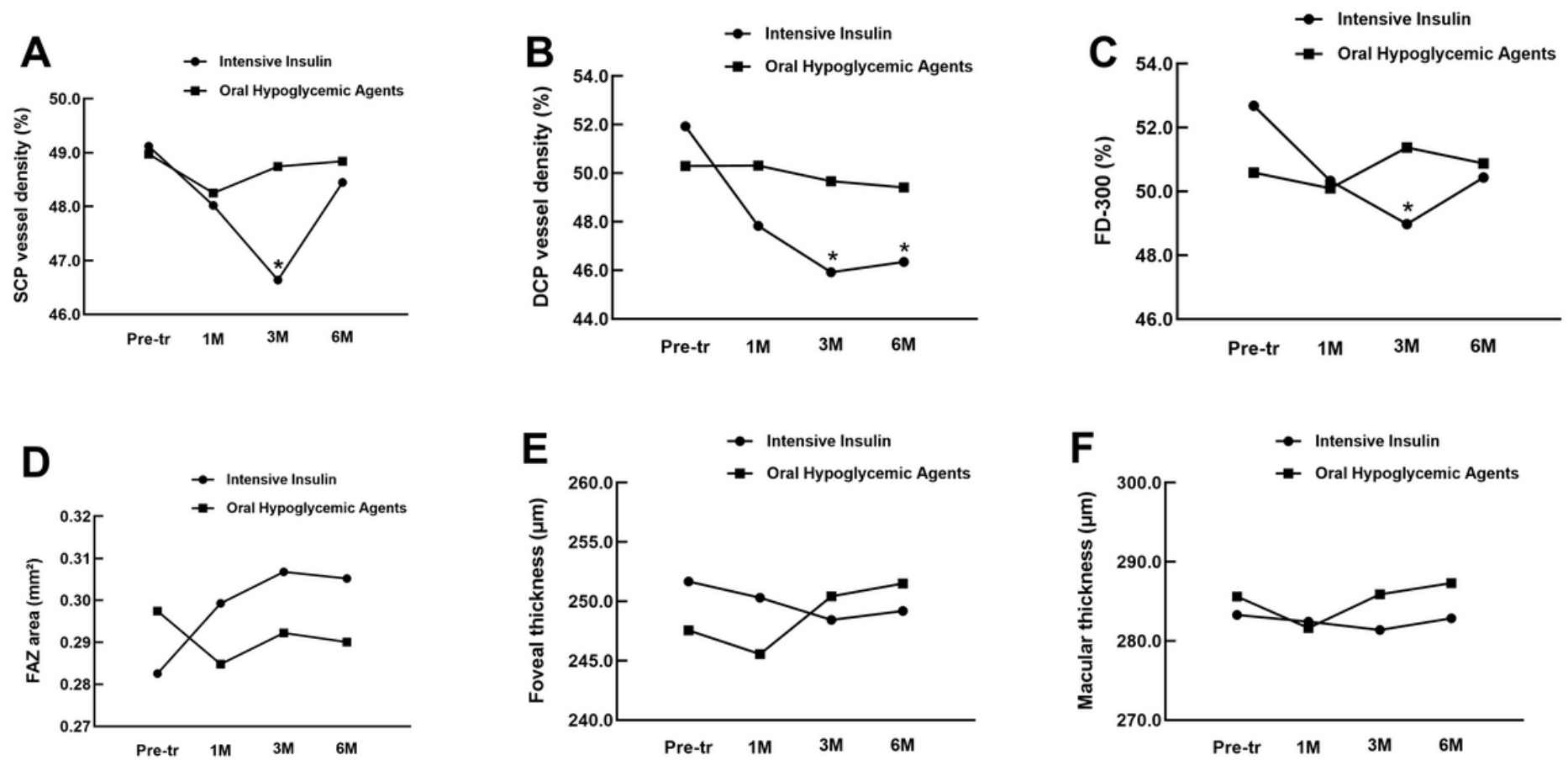

\section{Figure 1}

The longitudinal macular microvascular changes and macular thickness changes at pre-treatment, $1 \mathrm{M}, 3 \mathrm{M}$, and $6 \mathrm{M}$ following hypoglycaemic treatment in the intensive insulin group and oral hypoglycaemic agent group. The significant changes between the two groups compared at the same period points are marked with asterisks. a SCP vessel density changes; b DCP vessel density changes; c vessel density changes in FD-300; d FAZ area; e fovea thickness; $\mathbf{f}$ macular thickness. (Pre-tr = pre-treatment; $1 \mathrm{M}=$ at 1 month follow-up) 

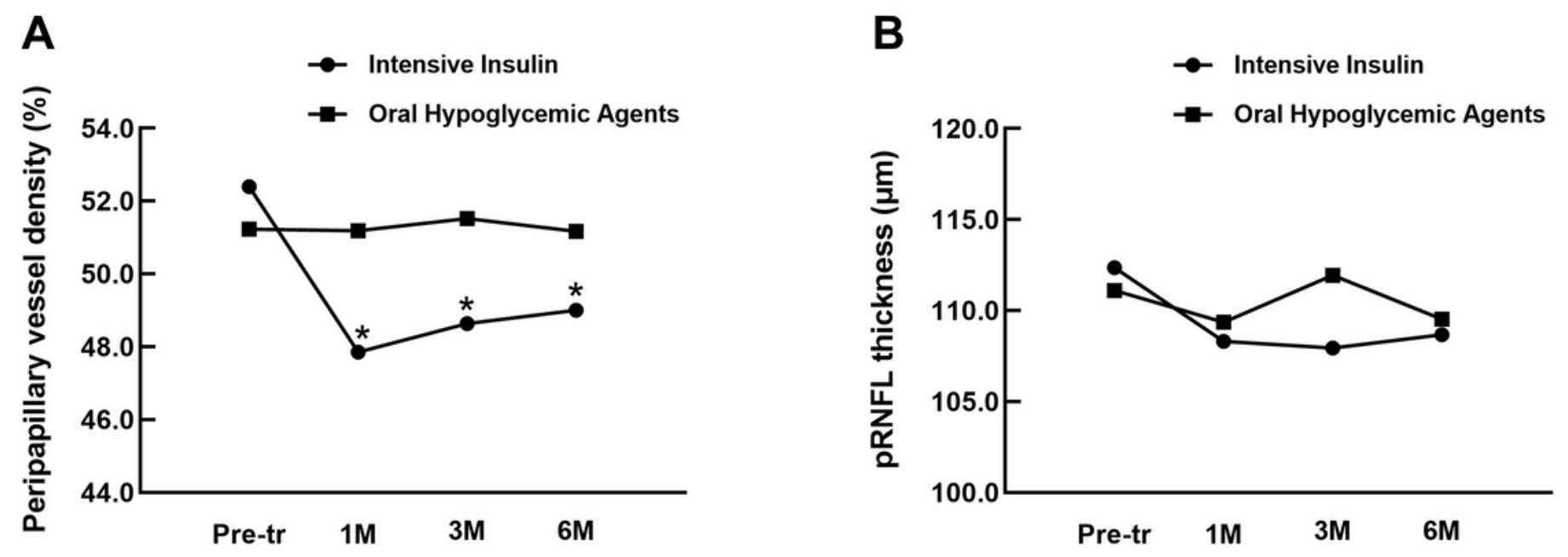

\section{Figure 2}

The longitudinal changes of ppVD and pRNFL at pre-treatment, $1 \mathrm{M}, 3 \mathrm{M}$, and $6 \mathrm{M}$ following hypoglycaemic treatment in the intensive insulin group and oral hypoglycaemic agent group. The significant changes between the two groups compared at the same period points are marked with asterisks. $\mathbf{a}$ ppVD changes; $\mathbf{b}$ pRNFL changes. (Pre-tr = pre-treatment; $1 \mathrm{M}=$ at 1 month follow-up)

\section{Supplementary Files}

This is a list of supplementary files associated with this preprint. Click to download.

- DataEffectsofintensiveinsulintherapyontheretinalmicrovasculatureinpatientswithtype2diabetesmellitus.xIsx 